\title{
Synthesis of some novel Heterocyclic Chalcone Derivatives
}

\section{Ahmed Hassan Shntaif}

\author{
Babylon university, College of science for women. Iraq \\ E-Mail: wsci.ahmed.hassan@uobabylon.edu.iq
}

Received:- 10/5/2017

Accepted:-17/8/2017

\section{Abstract:}

The derivatives of Chalcones were synthesized by reaction of 2,3-Dichlorobenzaldehyde with 2-Hydroxy acetophenone in the base medium, then the product react with thiourea, urea and hydrazine to preper the derivatives of thiazine, oxazine and isoxazole respectively. The structures of the synthesized compounds have been characterized by (IR, ${ }^{1} \mathrm{HNMR},{ }^{13} \mathrm{CNMR}$ and elemental analysis). Keywords: Chalcone, Urea, Claisen-Schmidt condensation, Oxazine and Isoxazole.

Chemistry Classifiication QD241-441 


\section{Introduction:}

Chalcones ( $\alpha, \beta$-unsaturated ketones) are prepared by condensing of acetophenone or its derivatives with aromatic aldehydes in basic medium, The structure of chalcone consists from two aromatic rings joined by ketoethylenic group ( $\mathrm{CO}-\mathrm{CH}=\mathrm{CH}-)$ [1]. Many methods are available for formation of carbon- carbon bonds in the synthesis of chalcones The

\section{Material and Methods:}

All the chemical and solvents used in the preparation of compounds were purchased from Sigma-Aldrich and Merch. All the reported melting points were determined using an electrically heated block with calibrated thermometer; samples were taken in open General procedure for synthesis of chalcone (1):

2,3-Dichlorobenzaldehyde $(0.1 \mathrm{~mol})$ and 2-Hydroxyacetophenone $(0.1 \mathrm{~mol})$ were dissolved in $(300 \mathrm{~mL})$ of ethanoic Sodium hydroxide $(10 \%)$ and the mixture was mixed for $(24 \mathrm{hrs})$ at $\mathrm{RT}$ then it was poured in $(400 \mathrm{~mL})$ of cold water with constant stirring and acidified with $10 \% \mathrm{HCl}$. The mixture left for (24 hrs) in Refrigerator. The solid product obtained was filtered, washed and recrystallized from ethanol. simplest method include Claisen-Schmidt condensation of equal molar quantities of acetophenone with aromatic aldehyde under an basic condition. Chalcones are also very useful intermediates for the synthesis of many important heterocyclic compounds. They are have five- [2,3], six- [2,4] and seven-rigs [5].

capillaries and were uncorrected. The IR Spectra were taken from Alpha Bruker IR spectrophotometer. ${ }^{1} \mathrm{HNMR}$ and ${ }^{13} \mathrm{CNMR}$ spectra were recorded on Bruker NMR (400 $\mathrm{MHz}$ ) in DMSO.

\section{Preparation of thiazine/ oxazine derivatives:}

A mixture of Chalcone (1) (0.2 mol), thiourea/ urea $(0.2 \mathrm{~mol})$ were dissolved in $(100 \mathrm{ml})$ of ethanolic sodium hydroxide, the mixture was left for (4 hrs) with stirring at RT. Then the mixture was poured into $(400 \mathrm{ml})$ of cold water with continuous stirring for an hour and then kept in refrigerator for ( $24 \mathrm{hrs})$. The precipitate obtained was filtered, washed and recrystallized. The completion of the reaction was monitored by TLC.

\section{Preparation the derivative of isoxazole:}

A mixture of Chalcone (1) $(0.2 \mathrm{~mol})$ and hydrazine hydrate $(0.2 \mathrm{~mol})$ were heated in 
tri ethyl amine $(50 \mathrm{ml})$ when the mixture began boiling $(5 \mathrm{~min})$ the heat was stopped. The mixture was left to cool and poured into cold water. The solid product was filtered, washed and recrystallized by ethanol.<smiles>CC(=O)c1ccccc1O</smiles><smiles>N#CC(O)CC1CCCCC1</smiles><smiles>O=C(/C=C/c1cccc(Cl)c1Cl)c1ccccc1O</smiles>

1

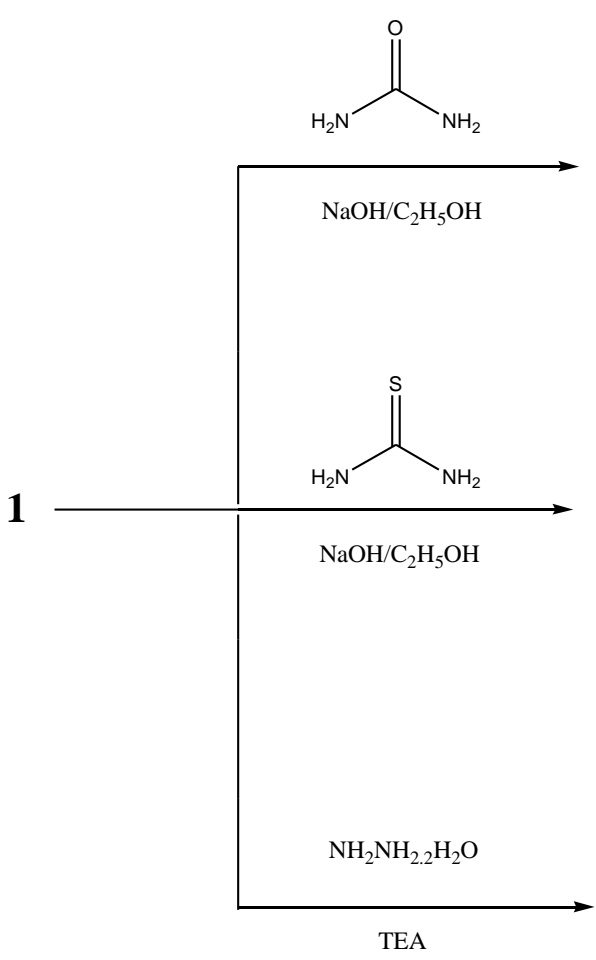<smiles>O=c1nc(-c2ccccc2O)cc(-c2cccc(Cl)c2Cl)[nH]1</smiles>

3<smiles>Oc1ccccc1NC1=NNC(c2cccc(Cl)c2Cl)C1</smiles>

\section{4}

Scheme 1. Synthetic procedure for preparation of title compounds.

\section{3-(2,3-dichlorophenyl)-1-(2-}

\section{hydroxyphenyl)prop-2-en-1-one (1):}

Yellow solid, M.p: $210 \mathrm{C}^{\mathrm{o}}$, Yield: 90\%, IR ( $v$ $\left.\mathrm{cm}^{-1}\right): 3350(\mathrm{O}-\mathrm{H}), 3071$ (=C-H), 2874, 2746 $(\mathrm{C}-\mathrm{H}$ aromatic $), 1733(\mathrm{C}=\mathrm{O}), 1683(\mathrm{C}=\mathrm{N}), 1557$ $(\mathrm{C}=\mathrm{C}),{ }^{1} \mathrm{H}$ NMR (DMSO): $\delta 2.64(\mathrm{~s}, \mathrm{H}, \mathrm{OH})$,
6.95-7.92 (m, 7H, $\left.\mathrm{H}_{\text {aromatic }}\right), 8.05-8.08(\mathrm{~d}, 2 \mathrm{H}$, $=\mathrm{C}-\mathrm{H}),{ }^{13} \mathrm{C}-\mathrm{NMR}(\mathrm{CDCl} 3): \quad \delta 193.4(\mathrm{C}=\mathrm{O})$, 161.9 (C-OH), 139.08 (=C-ph), 136.9, 135.1, 133.02, 132.06, 131.8, 130.01, 129.01, 128.08, 127.2, 121.08, 119.6, 118.2 (phenyl group). 
Anal. Calc. For $\mathrm{C}_{15} \mathrm{H}_{10} \mathrm{C}_{12} \mathrm{O}_{2} \mathrm{C}, 61.46$; H: 3.44. Found. C, 71.52; H, 5.24.

\section{6-(2,3-dichlorophenyl)-4-(2-}

\section{hydroxyphenyl)pyrimidin-2(1H)-one (2):}

Orang solid, M.p: $230 \mathrm{C}^{\mathrm{o}}$, Yield: 75\%, IR ( $v$ $\left.\mathrm{cm}^{-1}\right): 3310(\mathrm{O}-\mathrm{H}), 2991$ (=C-H), 2936, 2835 (C-H $\left.\mathrm{H}_{\text {aromatic }}\right), 1636(\mathrm{C}=\mathrm{O}), 1558(\mathrm{C}=\mathrm{C}) .{ }^{1} \mathrm{HNMR}$ (CDCl3): $2.5(\mathrm{~s}, \mathrm{H}, \mathrm{C}=\mathrm{C}-\mathrm{H}), 5.76(\mathrm{~s}, \mathrm{H}, \mathrm{OH})$, 6.99-7.84 (m, 7H, C-H aromatic), 8.04 ( N-H). ${ }^{13} \mathrm{C}-$ NMR (DMSO): 193.36 (N=C), 191.31 (C-N), 139.08, 136.9, 135.17, 133.08, 132.05, 131.4, $130.02,129.19,128.9,127.2,126.9,122.6$, 121.5, 119.6, 118.5 (phenyl group). 76.66 (=CH). Anal. Calc. For $\mathrm{C}_{16} \mathrm{H}_{10} \mathrm{Cl}_{2} \mathrm{~N}_{2} \mathrm{O}_{2}$ C, 57.68; H, 3.03; N, 8.41. Found. C, 61.47; H, 4.21; 9.78 .

\section{4-(2-hydroxybenzyl)-6-(2,3-}

dichlorophenyl)pyrimidine-2(1H)-thione (3): Brawn solid, M.p: $287 \mathrm{C}^{\mathrm{o}}$, Yield: $70 \%$, IR ( $v$ $\left.\mathrm{cm}^{-1}\right): 3432(\mathrm{O}-\mathrm{H}), 3018,(=\mathrm{C}-\mathrm{H}), 2935,2929$ (C-H $\left.\mathrm{H}_{\text {aromatic }}\right), 1648(\mathrm{C}=\mathrm{N}), 1399\left(\mathrm{C}=\mathrm{S}_{\mathrm{as}}\right), 753$ $\left(\mathrm{C}=\mathrm{S}_{\mathrm{s}}\right) .{ }^{1} \mathrm{H}$ NMR (DMSO): 2.25 (s, H, N-H),

\section{$\underline{\text { Results and Discussion: }}$}

\section{Spectroscopic characterization:}

\section{3-(2,3-dichlorophenyl)-1-(2}

hydroxyphenyl)prop-2-en-1-one was prepared as per Claisen-Schmidt condensation reaction which was converted to 6-(2,3-dichlorophenyl)4-(2-hydroxyphenyl)pyrimidin-2(1H)-one,4-(2hydroxybenzyl)-6-(2,3-dichlorophenyl) pyrimidine-2(1H)-thione and 2-(5-(2,3dichlorophenyl)-4,5-dihydro-1H-pyrazol-3- $4.83(\mathrm{~s}, \mathrm{H},=\mathrm{C}-\mathrm{H}), 5.25$ (s, H, O-H), 7.42-8.1 (m, 7H, $\left.\mathrm{CH}_{\text {aromatic }}\right) .{ }^{13} \mathrm{C}-\mathrm{NMR}$ (DMSO): 182 $(\mathrm{C}=\mathrm{S}), 178(=\mathrm{C}-\mathrm{NH}), 146(\mathrm{C}=\mathrm{N}), 142.5,142$, $136.5,133.5,133,130.5,126.5,126,121,120$, 110 (phenyl group), 104 (=C-H).

Anal. Calc. For $\mathrm{C}_{16} \mathrm{H}_{10} \mathrm{Cl}_{2} \mathrm{~N}_{2} \mathrm{OS}$ C, 55.04; $\mathrm{H}$, 2.90; N, 8.01; S, 9.16 Found. C, 60.14; H, 3.09; N,7.85; S, 10.63 .

\section{2-(5-(2,3-dichlorophenyl)-4,5-dihydro-1H- pyrazol-3-ylamino)phenol (4):}

Yellow-brawn solid, M.p:310 C', Yield: $75 \%$, IR $\left(v \mathrm{~cm}^{-1}\right): 3423(\mathrm{OH}), 3018,2935$ (C$\left.\mathrm{H}_{\text {aromatic }}\right), 2929\left(\mathrm{CH}_{2}\right), 1584(\mathrm{C}=\mathrm{N}),{ }^{1} \mathrm{H} \mathrm{NMR}$ (DMSO): 1.05 (d, 2H, $\left.\mathrm{CH}_{2}\right), 3.35-3.4$ (m, H, $\mathrm{CH}), 5.21$ (s, H, OH), 7.45-8.14 ( $\mathrm{m}, 7 \mathrm{H}$, $\left.\mathrm{H}_{\text {aromatic }}\right), 18.5(\mathrm{CH}), 44\left(\mathrm{CH}_{2}\right), 169.6(\mathrm{C}=\mathrm{N})$, 159, 152, 152.5, 146, 145, 134, 132.5, 131, 129, 126, 125, 123, 120.5, 114.5 (phenyl group). Anal. Calc. For $\mathrm{C}_{15} \mathrm{H}_{13} \mathrm{Cl}_{2} \mathrm{~N}_{3} \mathrm{O} \mathrm{C}, 55.92 ; \mathrm{H}$, 4.07; N, 13.04 Found. C, 58.47; H, 5.47; N, 15.14 .

ylamino) phenol by using urea, thiourea and hydrazine hydrate respectively. The IR spectra of compounds show absorption bands at (3310, 3350, 3423, 3432), (1648, 1584) and (1733, 1636) due to $\mathrm{OH}, \mathrm{C}=\mathrm{N}$ and $\mathrm{C}=\mathrm{O}$ groups respectively. The ${ }^{1} \mathrm{H}$ NMR spectra of compounds show a singlet at 2.64-5.21 attributed to $\mathrm{OH}$ protons. 
AL-Qadisiyah Journal of pure Science Vol.23 No. $2 \quad$ Year 2018

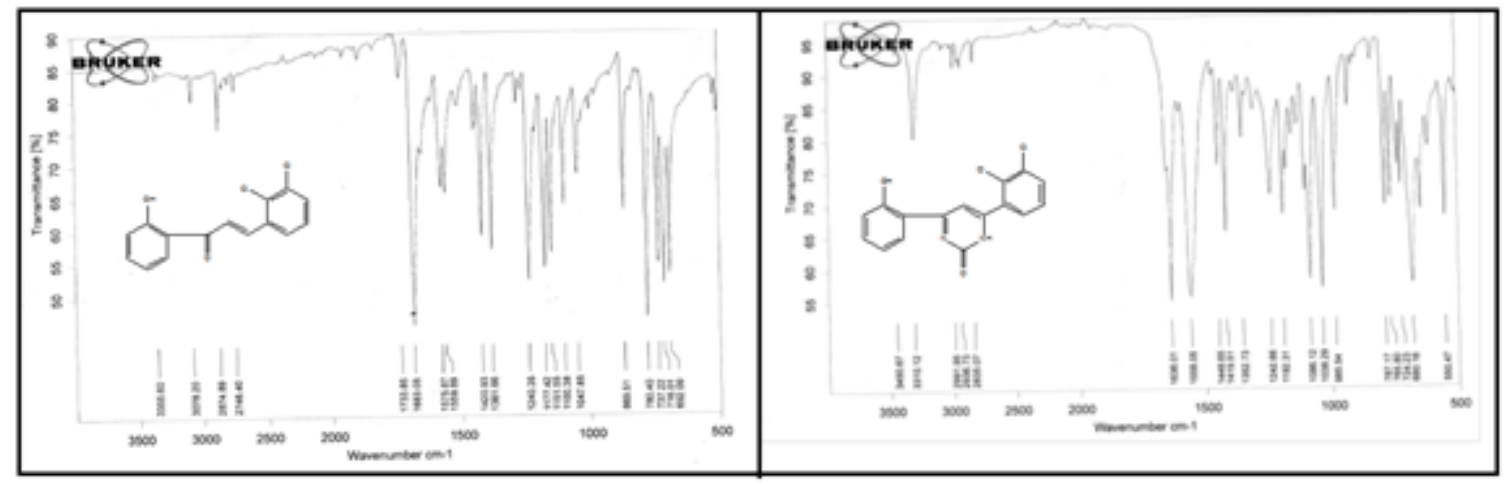

Fig l: IR spectra of compound l

Fig 2: IR spectra of compound 2

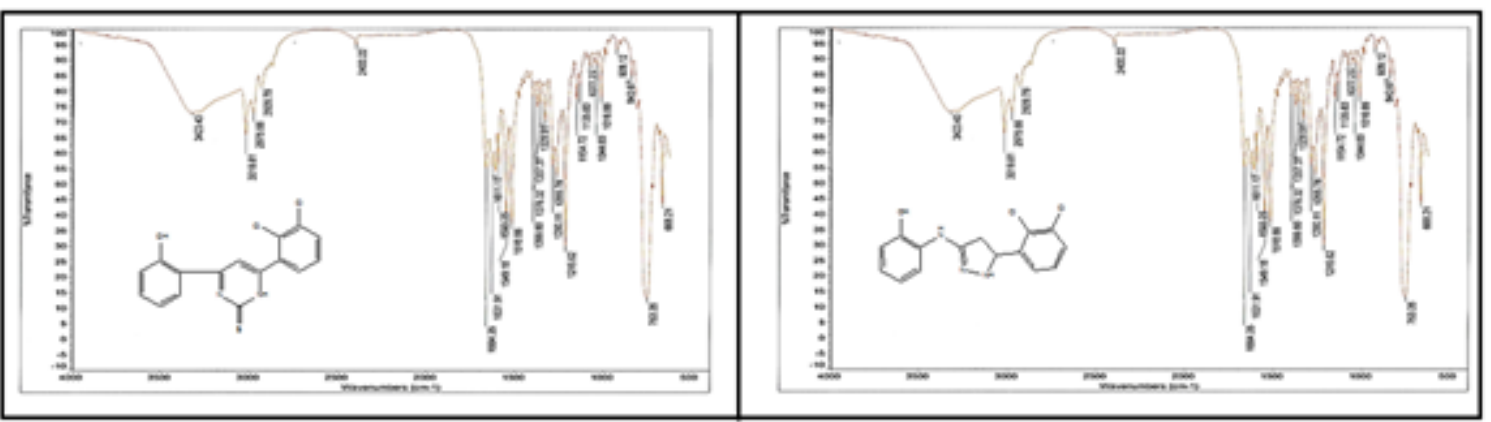

Fig l: IR spectra of compound 3

Fig 2: IR spectra of compound 4
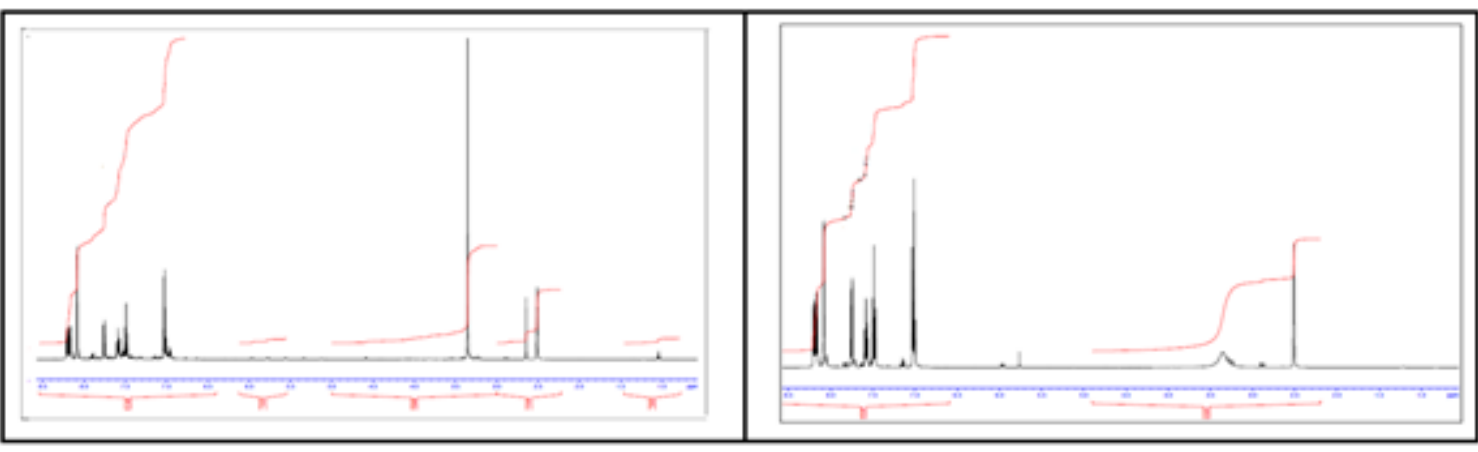

Fig 3: ${ }^{1}$ HNMR spectra of compound 1

Fig 4: ${ }^{1} \mathrm{HNMR}$ spectra of compound 2

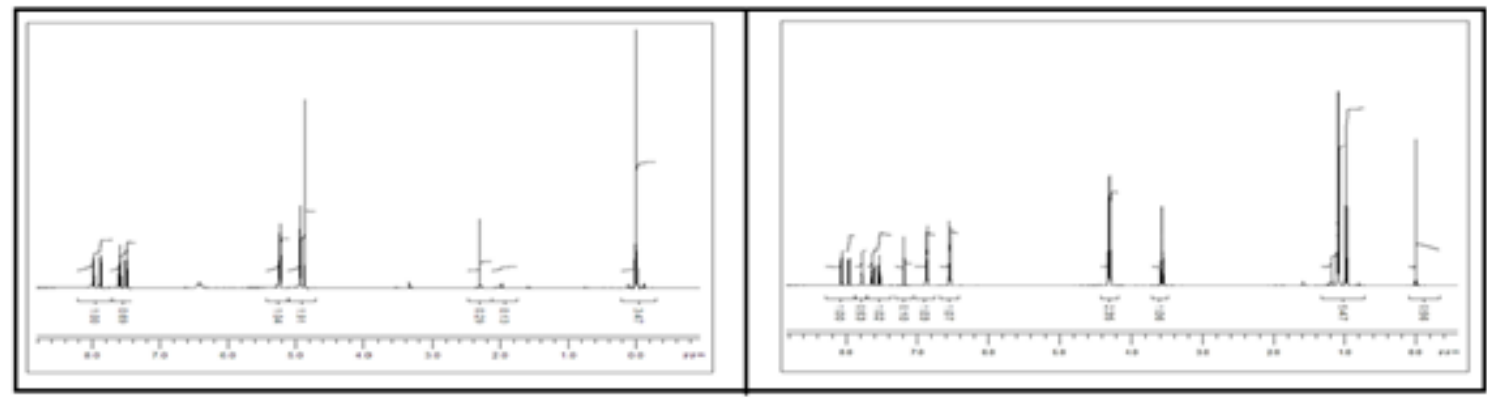

Fig 5: ${ }^{1} \mathrm{HNMR}$ spectra of compound 3

Fig 6: ${ }^{1} \mathrm{HNMR}$ spectra of compound 4 


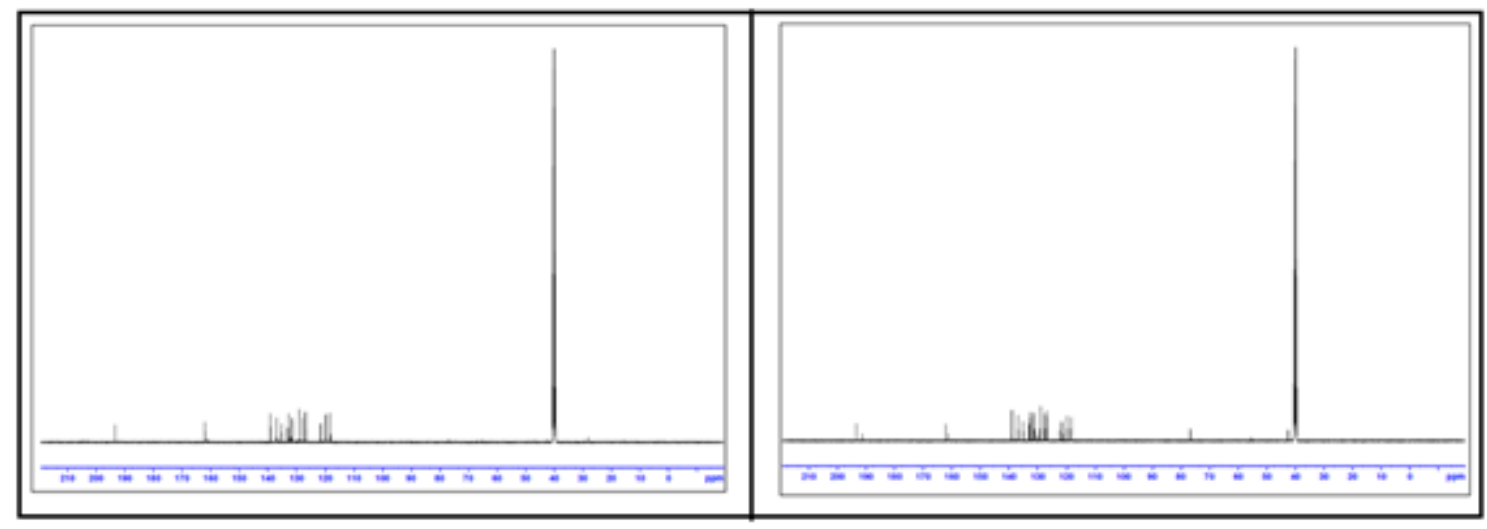

Fig 7: ${ }^{13}$ CNMR spectra of compound 1

Fig 8: ${ }^{13}$ CNMR spectra of compound 2

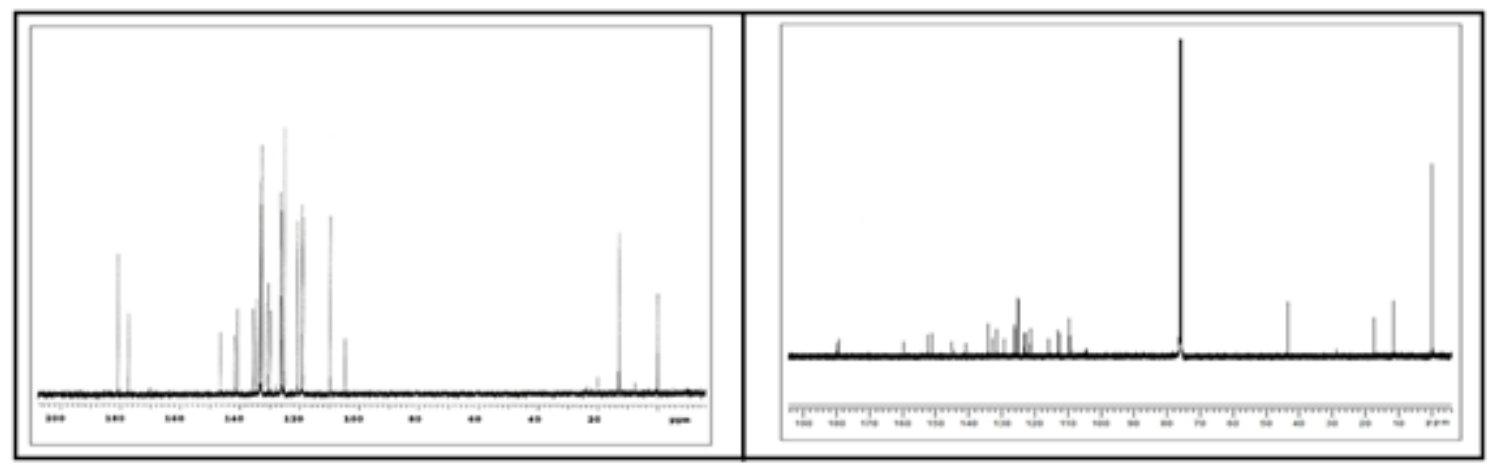

Fig 9: ${ }^{13}$ CNMR spectra of compound 3

Fig 10: ${ }^{13} \mathrm{CNMR}$ spectra of compound 4

\section{Conclusion:}

In summary, we have synthesized some novel hetero chalcones having oxazine, thiazine and isoxazole moiety. All the synthesized compounds gave satisfactory spectral and analytical data.

\section{Acknowledgement:}

The author is grateful to the University of Babylon, college of science for women in carrying out this research work.

\section{References:}

1- Sreedhar, N; Jayapal, M.; Sreenivasa, P. and Reddy, P. 2010. Synthesis and Characterization of 4-Hydroxy Chalcones Using PEG-400 as a Recyclable Solvent. RJPBCS, 1, 840845.
2- ABD El-Latif, N.; Amr, A. and Ibrahiem, A. 2007. Synthesis, reactions and pharmacological screening of heterocyclic derivatives using nicotinic acid as a natural synthon. Monatsh. Chem, 138, 559-567. 
3- Gaede, B. and Mcdermott, L.1993. Novel perfluoroalkyl-substituted pyrazoles. 1. hydroxypyrazoles. $J$. Heterocycl. Chem. 30, 49-54.

4- Shibata, K.; Katsuyama, I.; Izoe, H.; Matsui, M. and Muramatsu H. 1993. Synthesis of 4,6-di substituted 2-methyl pyridines and their 3-carboxamides. $J$. Heterocycl. Chem. 30, 277-281.

5- Jiaxi, X.; Wang, C. and Zhang, Q. 2001. Synthesis of 1-3,3a,5-tetraaryl3a,4,5,6-tetrahydro3 $H$-1,2,4 trizolo[4,3a][1,5] benzodiazepines. Heteroatom Chem. 6, 557-559.

6- Tomar, V.; Bhattacharjee, G.; Kamaluddina, and Ashok, K.2007. Synthesis and antimicrobial evaluation of new chalcones containing piperazine or 2,5-dichlorothiophene moiety, Bioorganic \& Medicinal Chemistry Letters 17, 5321-5324.

7- Anjani, S.; Kishor, K.; Ana, C.; Marina, S.; Irini, D. and Athina, G. 2010. Synthesis of some new S triazine based chalcones and their derivatives as potent antimicrobial agents, European Journal of Medicinal Chemistry, 45, 510-518.

8- Jalpa, C.; Jitender, B.; Kuldip, D.; Yogesh, T.; Sudhir, K.; Christophe, C.; Erik, D. and Anamik, K. 2007. Improved and rapid synthesis of new coumarinyl chalcone derivatives and their antiviral activity, Tetrahedron Letters, 48, 8472-8474.

9- Avnish, A. and Arvind, G. 2010. Synthesis and characterization of some pyrimidine-quinoline clubbed molecules and their microbicidal efficacy, Journal of Saudi Chemical Society, 14, 203-208.

10- Zhen-Hua, C.; Chang-Ji, Z.; LiangPeng, S. and Hu-Ri, P. 2010. Synthesis of new chalcone derivatives containing a rhodanine-3-acetic acid moiety with potential anti-bacterial activity, European Journal of Medicinal Chemistry, 45, 5739-5743. 
AL-Qadisiyah Journal of pure Science $\quad$ Vol.23 No. $2 \quad$ Year 2018

\section{تحضير بعض مشتقات الشُالكون الحلقية \\ احمد حسن شنتاف}

قسم الكيمياء، كلية العلوم للبنات، جامعة بابل.

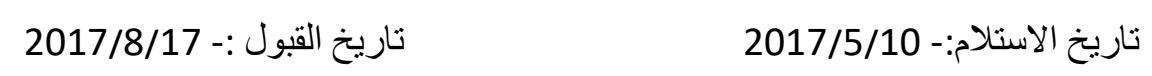

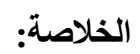

نم تحضير مشتقات الثالكون من خلال تفاعل 3,2- ثنائي كلورو بنز الدهايد مع 2-هيدروكسي اسيتوفينون في وسط قاعدي. ثم بعد ذلك

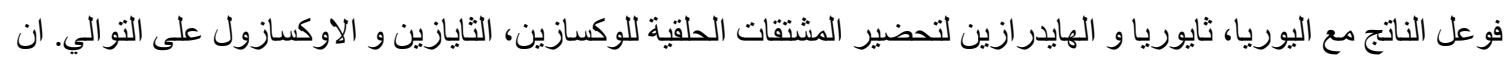
التركيب الكيميائي للمشتقات المحضرة نم تحديدها باستخدام مطيافية الاشعة تحت الحمر اءو، الرنين النووي المغناطيسي و تحليل

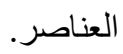

الكلمات المفتاحية: الثالكون، يوريا، تكاثف كليسن-شدت، الاوكسازين و الايزووكسازول. 